\title{
Global monopole in scalar-tensor theories of gravity
}

\author{
R. M. Teixeira Filho* \\ Departamento de Física, Universidade Federal da Paraíba, Caixa Postal 5008, 58059-970 João Pessoa, Pb, Brazil \\ and Instituto de Física, Universidade Federal da Bahia, Campus de Ondina, 40210-340 Salvador, Ba, Brazil \\ V. B. Bezerra ${ }^{\dagger}$ \\ Departamento de Física, Universidade Federal da Paraíba, Caixa Postal 5008, 58059-970 João Pessoa, Pb, Brazil
}

(Received 7 June 2001; published 28 August 2001)

\begin{abstract}
The gravitational field of a global monopole in the context of scalar-tensor theories of gravity is investigated. The spacetime is determined by solving the equation for the scalar field and using the global monopole solution of Barriola and Vilenkin, in the weak-field approximation. A comparison is made with the results predicted by Brans-Dicke theory and general relativity.
\end{abstract}

DOI: $10.1103 /$ PhysRevD.64.067502

\section{INTRODUCTION}

Topological defects may arise in gauge models with spontaneous symmetry breaking. They can be of various types, such as monopoles, domain walls, strings, and their hybrids $[1,2]$. Their nature depends on the topology of the vacuum manifold of the field theory under consideration.

Among the topological defects mentioned previously, in this paper we will focus on global monopoles. The simplest model that gives rise to a global monopole is described by a system composed of a triplet of isoscalar fields whose original global $\mathrm{O}(3)$ gauge symmetry was spontaneously broken to $\mathrm{U}(1)$.

The solution corresponding to a global monopole in an $\mathrm{O}$ (3) broken symmetry model has been investigated by Barriola and Vilenkin [3] in the context of general relativity.

The energy-momentum tensor of a global monopole configuration in regions far away from the core can be approximated by

$$
\widetilde{T}_{t}^{t}=\widetilde{T}_{r}^{r}=\frac{\eta^{2}}{r^{2}}, \quad \widetilde{T}_{\theta}^{\theta}=\widetilde{T}_{\varphi}^{\varphi}=0
$$

It is worth noticing that far from the global monopole core, the main effects are produced by the deficit solid angle, thus we can neglect the mass term, and the respective metric in Einstein's theory of gravity can be written as [3]

$$
\begin{aligned}
d s^{2}= & \left(1-8 \pi G \eta^{2}\right) d T^{2}-\left(1-8 \pi G \eta^{2}\right)^{-1} d R^{2}-R^{2}\left(d \theta^{2}\right. \\
& \left.+\sin ^{2} \theta d \varphi^{2}\right),
\end{aligned}
$$

where $G$ is the Newtonian gravitational constant and $\eta$ is the energy scale of symmetry breaking.

Let us consider the weak-field approximation. Then, in this case, metric coefficients should be computed up to first order in $G \eta^{2}$, and the line element reads

\footnotetext{
*Electronic address: rmuniz@ fisica.ufpb.br

†Electronic address: valdir@ fisica.ufpb.br
}

PACS number(s): 04.50. + h

$$
\begin{aligned}
d s^{2}= & \left(1-8 \pi G \eta^{2}\right) d T^{2}-\left(1+8 \pi G \eta^{2}\right) d R^{2}-R^{2}\left(d \theta^{2}\right. \\
& \left.+\sin ^{2} \theta d \varphi^{2}\right) .
\end{aligned}
$$

If we introduce the coordinate transformation

$$
\begin{aligned}
& t=\left(1-4 \pi G \eta^{2}\right) T, \\
& r=\left(1+4 \pi G \eta^{2}\right) R,
\end{aligned}
$$

it is easy to see that line element given by Eq. (3) assumes the form

$$
d s^{2}=d t^{2}-d r^{2}-b^{2} r^{2}\left(d \theta^{2}+\sin ^{2} \theta d \varphi^{2}\right),
$$

where $b^{2}=1-8 \pi G \eta^{2}<1$.

This metric corresponds to a spacetime with a deficit solid angle $\Delta=32 \pi^{2} G \eta^{2}$ : test particles are deflected (topological scattering) by an angle $\pi(\Delta / 2)$ irrespective of their velocity and impact parameter. In spite of having constant coefficients $g_{00}$ and $g_{r r}$, this metric represents a curved spacetime whose curvature vanishes in the case $b=1$ (flat spacetime). For $\theta$ $=\pi / 2$, the metric (5) is exactly the same as that of a gauge cosmic string, in which case the azimuthal angle $\varphi$ has a deficit $\Delta=2 \pi(1-b)$. Therefore, the gravitational field of a global monopole exhibits some interesting properties, particularly those concerning the appearance of nontrivial spacetime topologies.

The scalar-tensor theory of gravity was proposed some years ago [4] and represents a generalization of the simplest scalar-tensor theory of gravity, namely the Brans-Dicke theory [5]. In general scalar-tensor theories of gravity, the gravitational field is not described only by the usual tensor field $g_{\mu \nu}$ of general relativity. In addition, we have one or several long-range scalar fields that also mediate gravitational interaction.

Scalar-tensor theories of gravity have been a subject of renewed interest. Certainly, one motivation for this is the belief that, at least at sufficiently high-energy scales, gravity becomes scalar-tensorial in nature [6] and therefore these theories are important in the very early Universe. On the other hand, two important theoretical developments have been achieved in, for example, unification models based on superstrings that naturally associate long-range scalar part- 
ners to the usual tensor gravity of Einstein [7]. Another motivation for the investigation of scalar-tensor theories is that inflationary cosmology in this framework seems to solve the fine-tuning problem and in this way gives us a mechanism of terminating inflationary eras [8].

Apart from the solution of this problem, the scalar-tensor theories by themselves have direct implications for cosmology and for experimental tests of gravitational interaction [9], and they have importance in the early Universe in which scenario topological defects may have been produced. Therefore, it is important to consider the topological defects in this context, and for this reason some authors have obtained gravitational fields of cosmic strings and domain walls [10] and the global monopole [11,12] in the Brans-Dicke theory of gravity. Also, solutions corresponding to a cosmic string [13] and a domain wall [14] in more general scalar-tensor theories of gravity have been obtained.

In this paper, we consider the global monopole and investigate its gravitational field in the scalar-tensor theories of gravity using the weak-field approximation. Our approach consists of working out the field equation corresponding to the scalar field (dilaton), determining its solution, and then incorporating it with the global monopole solution of general relativity [3], with the appropriate modifications, in the weak approximation in order to construct the solution corresponding to the global monopole in scalar-tensor theories.

\section{THE METRIC OF A GLOBAL MONOPOLE IN THE WEAK-FIELD APPROXIMATION}

In this section, we will derive the metric of a global monopole in the framework of massless scalar-tensor theories. Let us consider the action describing the class of scalartensor theories developed in Ref. [4]. In the so-called Einstein (conformal) frame, the action from which the global monopole solution is obtained reads

$$
\begin{aligned}
S= & \frac{1}{16 \pi G_{*}} \int d^{4} x \sqrt{-g}\left[R-2 g^{\mu \nu} \partial_{\mu} \phi \partial_{\nu} \phi\right] \\
& +\int d^{4} x \sqrt{-g} A^{2}(\phi)\left[\frac{1}{2} g^{\mu \nu} \partial_{\mu} \Phi \partial_{\nu} \Phi-V(\Phi)\right],
\end{aligned}
$$

where $g_{\mu \nu}$ is a pure rank-2 metric tensor, $R$ is the curvature scalar associated to it and $G_{*}$ is some "bare" gravitational coupling constant. The second term on the right-hand side of Eq. (6) is the matter action representing a model of a real Higgs scalar field $\Phi$, and $V(\Phi)$ is the symmetry-breaking potential. Action (6) can be obtained from the original action that appears in Ref. [4] by a conformal transformation (see, for instance, [15]),

$$
\tilde{g}_{\mu \nu}=A^{2}(\phi) g_{\mu \nu},
$$

where $\tilde{g}_{\mu \nu}$ is the physical metric and contains both scalar and tensor degrees of freedom and $A^{2}(\phi)$ is an arbitrary function of the scalar field.

In the Einstein frame, the field equations are written as follows:

$$
\begin{aligned}
& R_{\mu \nu}=2 \partial_{\mu} \phi \partial_{\nu} \phi+8 \pi G_{*}\left(T_{\mu \nu}-\frac{1}{2} g_{\mu \nu} T\right), \\
& \square_{g} \phi=-4 \pi G_{*} \alpha(\phi) T,
\end{aligned}
$$

where $\alpha(\phi) \equiv[\partial \ln A(\phi)] / \partial \phi$, which can be interpreted as the (field-dependent) coupling strength between matter and the scalar field, and the energy-momentum tensor is obtained from

$$
T^{\mu \nu} \equiv \frac{2}{\sqrt{-g}} \frac{\delta S_{m}}{\delta g_{\mu \nu}} .
$$

In what follows, we will consider the solution of a global monopole in the weak-field approximation. Therefore, we will expand Eqs. (8) to first order in $G_{*} A^{2}\left(\phi_{0}\right)$ in such a way that

$$
\begin{aligned}
g_{\mu \nu} & =\eta_{\mu \nu}+h_{\mu \nu}, \\
\phi & =\phi_{0}+\phi_{(1)}, \\
A(\phi) & =A\left(\phi_{0}\right)\left[1+\alpha\left(\phi_{0}\right) \phi_{(1)}\right], \\
T_{\nu}^{\mu} & =T_{(0) \nu}^{\mu}+T_{(1) \nu}^{\mu},
\end{aligned}
$$

where $\phi_{0}$ denotes a determined value of the scalar field.

In this approximation,

$$
T_{(0) \mu \nu}=A^{2}\left(\phi_{0}\right) \widetilde{T}_{(0) \mu \nu}
$$

is the energy-momentum tensor of a scalar-tensor global monopole, where $\widetilde{T}_{(0) \nu}^{\mu}$ is the energy-momentum tensor of a global monopole in general relativity and is given by Eq. (1).

In the linearized regime, Eqs. (8) can be written as

$$
\nabla^{2} h_{\mu \nu}=16 \pi G_{*}\left(T_{(0) \mu \nu}-\frac{1}{2} \eta_{\mu \nu} T_{(0)}\right)
$$

and

$$
\nabla^{2} \phi_{(1)}=4 \pi G_{*} \alpha\left(\phi_{0}\right) T_{(0)} .
$$

Let us begin by solving the equation for the dilaton field $\phi_{(1)}$ in Eq. (13). We have that

$$
\nabla^{2} \phi_{(1)}=8 \pi G_{0} \alpha\left(\phi_{0}\right) \frac{\eta^{2}}{r^{2}}
$$

whose solution is

$$
\phi_{(1)}=8 \pi G_{0} \alpha\left(\phi_{0}\right) \ln \frac{r}{r_{0}}+\frac{2 M \alpha^{2}\left(\phi_{0}\right)}{r},
$$

where $G_{0} \equiv G_{*} A^{2}\left(\phi_{0}\right)$ is a $\phi_{0}$-dependent effective gravitational constant, and $r_{0}$ and $2 M \alpha^{2}\left(\phi_{0}\right)$ are integration constants, $M$ being the mass of the monopole core. This last integration constant was obtained in analogy with the procedure done in Ref. [11]. 
Now, the linearized Einstein equation in Eq. (12) with the source given by Eq. (1) multiplied by the factor $A^{2}(\phi)$ is just the same as in general relativity with $G$ changed by $G_{0}$ $=\left\{1 /\left[1+\alpha^{2}\left(\phi_{0}\right)\right]\right\} G$. This relation between $G$ and $G_{0}$ was derived taking into account the corresponding one in BransDicke theory, in which case this relation is valid for $\alpha^{2}$ $=1 /(2 \omega+3)$. Then, in order to construct the solution that corresponds to a global monopole in scalar-tensor theories of gravity, we can use the linearized form of the Barriola and Vilenkin [3] solution given by Eq. (5) with $G$ interchanged by $G_{0}=\left\{1 /\left[1+\alpha^{2}\left(\phi_{0}\right)\right]\right\} G$, together with Eq. (15), in which we drop the mass term as it is totally negligible on the astrophysical scale. Doing this we have that, in the weak-field approximation, the metric that represents the gravitational field of a scalar-tensor global monopole reads

$$
\begin{aligned}
d s^{2}= & A^{2}\left(\phi_{0}\right)\left[1+16 \pi \frac{\alpha^{2}\left(\phi_{0}\right)}{1+\alpha^{2}\left(\phi_{0}\right)} G \eta^{2} \ln \left(\frac{r}{r_{0}}\right)\right]\left[d t^{2}-d r^{2}\right. \\
& \left.-\left(1-\frac{8 \pi}{1+\alpha^{2}\left(\phi_{0}\right)} G \eta^{2}\right) r^{2}\left(d \theta^{2}-\sin ^{2} \theta d \phi^{2}\right)\right],
\end{aligned}
$$

where we have used the fact that the physical metric, in this approximation, is given by

$$
\tilde{g}_{\mu \nu}=A^{2}\left(\phi_{0}\right)\left[1+2 \alpha\left(\phi_{0}\right) \phi_{(1)}\right]\left(\eta_{\mu \nu}+h_{\mu \nu}\right) .
$$

The factor $A^{2}$ ( $\left.\phi_{0}\right)$ appearing in the above expression can be absorbed by a redefinition of the coordinates $(t, r, \theta, \phi)$. We obtain finally

$$
\begin{aligned}
d s^{2}= & {\left[1+16 \pi \frac{\alpha^{2}\left(\phi_{0}\right)}{1+\alpha^{2}\left(\phi_{0}\right)} \eta^{2} \ln \left(\frac{r}{r_{0}}\right)\right]\left[d t^{2}-d r^{2}-(1\right.} \\
& \left.\left.-\frac{8 \pi}{1+\alpha^{2}\left(\phi_{0}\right)} G \eta^{2}\right) r^{2}\left(d \theta^{2}-\sin ^{2} \theta d \phi^{2}\right)\right] .
\end{aligned}
$$

Thus, we have shown that in the weak-field approximation, Eq. (18) represents the spacetime generated by a global monopole in scalar-tensor theories of gravity. Analogously to the general relativity and Brans-Dicke cases, this curved spacetime also presents a deficit solid angle in the hypersurface $t=$ const. The area of a sphere of radius $r$ in this space would be given by

$$
4 \pi r^{2}\left[1-\frac{8 \pi \eta^{2} G}{1+\alpha^{2}\left(\phi_{0}\right)}+16 \pi \frac{\alpha^{2}\left(\phi_{0}\right)}{1+\alpha^{2}\left(\phi_{0}\right)} \eta^{2} G \ln \left(\frac{r}{r_{0}}\right)\right],
$$

rather than $4 \pi r^{2}$. Therefore, the deficit solid angle becomes $\phi_{0}$-dependent as compared with the result in general relativity.

Concerning the light deflection in the spacetime of a scalar-tensor global monopole, one can follow the arguments of Barriola and Vilenkin [3], also used by Barros and Romero [11] in the context of Brans-Dicke theory, to show that a light signal propagating from a source $S$ to an observer $O$, when $S, O$, and the monopole are perfectly aligned, produces an image with the form of a ring of angular diameter given by

$$
d \Omega=\frac{8 \pi \eta^{2} G}{1+\alpha^{2}\left(\phi_{0}\right)} \frac{l}{l+d},
$$

where $d$ and $l$ are the distances from the monopole to the observer and to the source, respectively. This result reduces to those obtained in the framework of general relativity and Brans-Dicke theory in the appropriate limit, as it should be.

\section{CONCLUDING REMARKS}

In this work, we have extended the monopole solution in the context of general relativity [3] to scalar-tensor theories of gravity. Naturally, from our results, the corresponding solution [11] in the context of Brans-Dicke theory as well as the solution in general relativity [3] are reobtained in the appropriate limit, as was already pointed out. For example, we can obtain the solution [11] in Brans-Dicke theory by taking $\alpha^{2}=1 /(2 \omega+3)$, where $\omega$ is a dimensionless coupling constant. The solution in general relativity [3] is obtained by taking $\alpha \rightarrow 0$, which is equivalent to taking [16] $\omega \rightarrow \infty$.

The method we have used is simpler than the one used in [11], which worked out a set of Brans-Dicke equations. In the present case, we just solved explicitly the equation for the scalar field (dilaton) and used the argument that the solution of the equation for $h_{\mu \nu}$ can be obtained directly from the global monopole solution in general relativity, interchanging the Newtonian gravitational constant by the $\phi_{0^{-}}$ dependent effective gravitational constant $G_{0}=\{1 /[1$ $\left.\left.+\alpha^{2}\left(\phi_{0}\right)\right]\right\} G$. This solution can be used, in principle, to study some effects due to geometrical and topological features of spacetime, thereby providing ways to compare with the corresponding results in general relativity and BransDicke theory.

\section{ACKNOWLEDGMENTS}

We are indebted to C. Romero and M. E. X. Guimarães for enlightening discussions. This work was partially supported by CNPq (Brazilian Agency).
[1] T.W.B. Kibble, J. Phys. A 9, 1387 (1976).

[2] E. P. S. Shellard and A. Vilenkin, Cosmic Strings and Other Topological Defects (Cambridge University Press, Cambridge, England, 1994).

[3] M. Barriola and A. Vilenkin, Phys. Rev. Lett. 63, 341 (1989). [4] P.G. Bergmann, Int. J. Theor. Phys. 1, 25 (1968); R.V. Wag- oner, Phys. Rev. D 1, 3209 (1970); K. Nordtvedt, Jr., Astrophys. J. 161, 1059 (1970).

[5] C. Brans and R.H. Dicke, Phys. Rev. 124, 925 (1961).

[6] Thibault Damour and A. Polyakov, Nucl. Phys. B423, 532 (1994); Gen. Relativ. Gravit. 26, 1171 (1994).

[7] M. B. Green, J. H. Schwarz, and E. Witten, Superstring Theory 
(Cambridge University Press, Cambridge, England, 1987).

[8] D. La and P.J. Steinhardt, Phys. Rev. Lett. 62, 376 (1989); P.J. Steinhardt and F.S. Acceta, ibid. 64, 2740 (1990); J. Garcia Belido and M. Quiros, Phys. Lett. B 243, 45 (1990); J.D. Barrow and K. Maeda, Nucl. Phys. B341, 294 (1990).

[9] Thibault Damour, Nucl. Phys. B (Proc. Suppl.) 80, 41 (2000).

[10] A. Barros and C. Romero, J. Math. Phys. 36, 5800 (1995).

[11] A. Barros and C. Romero, Phys. Rev. D 56, 6688 (1997).

[12] A. Banerjee, A. Beesham, S. Chatterjee, and A.A. Sen, Class.
Quantum Grav. 15, 645 (1998).

[13] M.E.X. Guimarães, Class. Quantum Grav. 14, 435 (1997).

[14] V.B. Bezerra, L.P. Colatto, M.E.X. Guimarães, and R.M. Teixeira Filho, gr-qc/0104038.

[15] Thibault Damour and K. Nordtvedt, Phys. Rev. Lett. 70, 2217 (1993); Phys. Rev. D 48, 3436 (1993).

[16] For a discussion concerning this limit, see C. Romero and A. Barros, Phys. Lett. A 173, 243 (1993). 